Fountain Journal of Natural and Applied Sciences: 2018; 7(1): 1 - 11
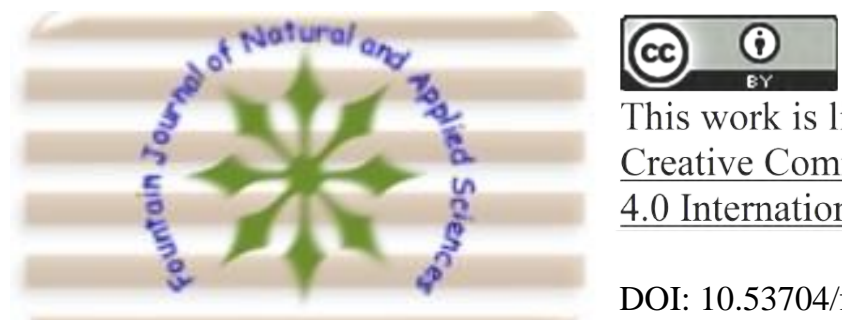

This work is licensed under

Creative Commons Attribution

4.0 International License.

DOI: 10.53704/fujnas.v7i1.169

A publication of College of Natural and Applied Sciences, Fountain University, Osogbo, Nigeria.

Journal homepage: www. fountainjournals.com

ISSN:2354-337X(Online),2350-1863(Print)

\title{
A Qualitative Study of Signal Strength Coverage of Digital Terrestrial Television in Ibadan South Western Nigeria
}

\author{
*Bolarinwa, H. S., Ademola O. D., Yusuf, A. and Animasahun, L. O.
}

Department of Physics, Electronics and Earth Sciences, Fountain University, Osogbo

\begin{abstract}
This work investigated the quality of signal strength coverage of the existing Digital Terrestrial Television (DTTV) operator in Nigeria using Startimes Ibadan as a case study. Signal strength and altitude data at different locations within a $40 \mathrm{~km}$ radius of the Startimes transmitting station were measured to determine the quality of signal strength reception of Startime's digital television in Ibadan metropolis. A signal analyzer, a 10-meter cable, Yaggi external antenna and Global Positioning System (GPS) were used for the measurements at various locations in Ibadan within $40 \mathrm{~km}$ radius distance of Startimes based station at Agodi in Ibadan. The city was grouped into six different zones for proper signal testing and efficient data0 collation. Parameters such as signal strength, distance, altitude, signal to noise ratio, modulation error rate, pre-bit error rate and post bit-error rate were measured and recorded. The effects of distance and altitude were analyzed. It was observed from the result that there is a direct relationship between power level and altitude. In addition, there is an inverse relationship between power level and distance.
\end{abstract}

Keywords: DTTV, Signal Strength, Altitude, Distance, DVB-T

\section{Introduction}

Digital Video Broadcasting Television (DVB-T) came up as the first system based on Orthogonal Frequency Division Multiplexing (OFDM) modulation for digitalization of television when considering terrestrial propagation in the early 90's (Satitsamitpong and Mitomo, 2013). DVB-T is an improvement to the analogue system, the robustness of its modulation eliminates the limitation and shortcoming encountered in the analogue system, and the technology was proposed by the DVB Consortium and standardized by the European Telecommunication Standard Institute (ETSI) (Dhok and Dhanvijay, 2015; Gyamfi, 2015). A digital terrestrial television (DTTV) is a terrestrial implementation of Digital Television (DTV) technology that uses an aerial to broadcast to a television antenna or aerial instead of satellite dish or cable television technology (Seamus, 2000; Adda and Ottaviani, 2005). DTTV is usually transmitted on radio frequencies through terrestrial space in the same way a standard analogue television is transmitted (Ajibola, 2015). However, it uses the multiplex transmitter to allow reception of multiple channels on a single frequency range known as a subchannel. This enables television stations to provide clearer pictures and better sound quality (Telecom Regulatory Authority of India, 2017).

*Corresponding author: +2348056668594

Email address: babdulhakeem@gmail.com 
DTTV transmits information such as picture and sound as "data bits" like a computer. It also enables a television station to deliver a high quality television services for consumers with high definition (HD) television sets and offer such essential data services such as: closed-captioning, electronic program guides, additional languages (spoken or subtitled), and allow other advanced services such as live broadcasting and interactivity functionality (Collins, 2001; Balarabe, 2013; BT.2140-7, 2014).

Terrestrial Television services brought many advantages and benefits to viewers compared to conventional analogue. Although many countries around the world introduced DTTV with the attendant benefits, it provides in mind (Simon, 2000; Plum, 2014). The rates of DTTV adoption have been slow, and many a time some countries have to postpone the analogue switch-off date, including Nigeria (Idachaba, 2018).

Digital Terrestrial Television (DTTV) migration have a great deal of complexity, which bound up with many issues such as technical, social and economic. For any change in technology, there has to be a considerable challenges attached to it. Identifying, monitoring and mitigating these challenges are therefore, necessary (Mbatha and Lesame, 2014; Nkaka and Mukumbwa, 2016).

In compliance with the International Telecommunications Union, ITU directive to migrate from analogue to digital television transmission, the National Broadcasting Commission which is the body responsible for broadcasting regulation in Nigeria, recognizes, digitization as an important global movement and believed that the transition to digital terrestrial broadcasting will affect all segments along the broadcasting value chain positively (Idoko, 2010; NBC, 2018)

Nigeria and the rest of the world have experienced a dramatic rise in the use of television (TV) sets and has even become essential part of everyday life of their citizens. The introduction of many operators and competitive technologies has driven the price of DTTV downwards, thereby compelling more users across the social divide and age to subscribe to the services. (Idachaba, 2018)

In December 2007, the National Broadcasting Commission (NBC) set June 17, 2012, for the complete switchover from analogue TV to digital television. This date was shifted to June 2015 and later to June 17,2017 , due to unforeseen challenges that hinder the implementation (NBC, 2016)

According to Davis, (2009); Satitsamitpong and Mitomo (2013), there are needs to examine and understand the factors affecting the quality of signal reception of DTTV so that the intention of switching over from analogue to digital television will not be defeated. As Nigeria is switching from analogue transmission to digital transmission, there is the need to study the quality of the signal strength coverage of the existing DTTV operator in the country and determine if they are in line with the standards set by the ITU for the DTTV services.

In this work, we investigated the signal coverage quality within a $40 \mathrm{~km}$ radius of transmitting site of Startimes. The effect of distance and altitude on the signal power strength were analyzed and determine if the transmitter parameters such as bit error rate, Modulation error rates of Startimes are within the specified standards.

\section{Materials and Method}

The material used for the collation of data include, a DS2400T DVB-T2 Signal analyzer meter, a Global Positioning System (GPS), 10 meter high external yaggi antenna connected with the signal analyzer and mounted as shown in Figure 1, and a car for transportation from one location to the other. The Startimes transmitting base is located inside the national television authority (NTA) premises at Agodi gate in Ibadan city on latitude $7^{\circ} 23^{\prime} 45.738^{\prime \prime} \mathrm{N}$ and Longitude $3^{\circ} 55^{\prime} 2.742^{\prime \prime} E$. The station is at altitude 252.40 meter above the sea level. Figure 2 presents the map of the study area.

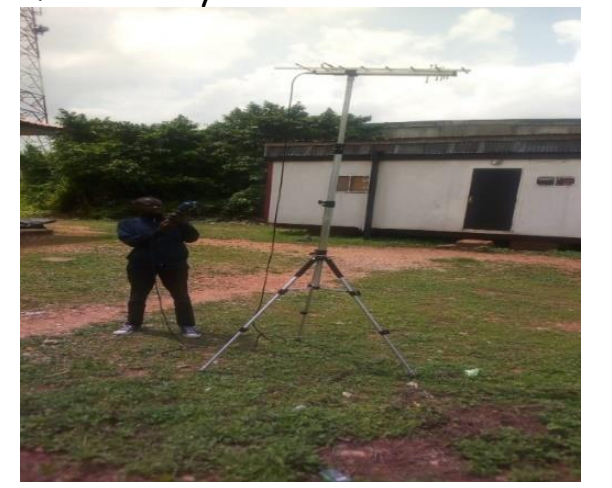

Figure 1. Yagi receiving antenna set up 


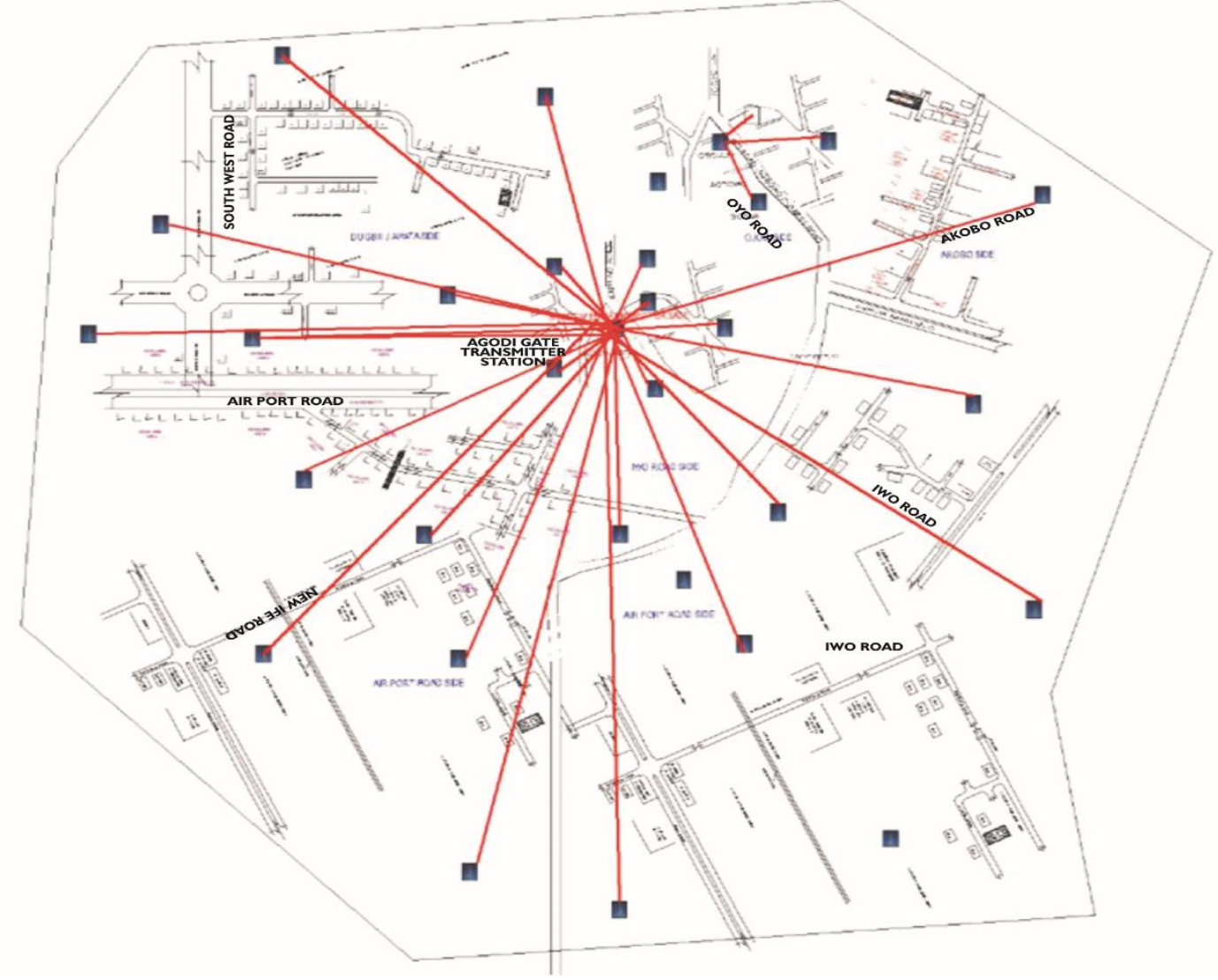

Figure 2. Ibadan city map indicating various zone of signal testing location and data collation

Signals from two transmitting antennas radiating at frequencies of $738 \mathrm{~Hz}$ and $762 \mathrm{~Hz}$ with a transmitted power of $2.5 \mathrm{~kW}$ were monitored from Oyo Road, Akobo Road, Iwo Road, New Ife Road, Southwest Road, and Airport Road in Ibadan. The radiating antennas were mounted on a transmitting mast height at $250 \mathrm{ft}$ at the Startimes transmitting base in Agodi. Several readings such as signal strength (SS), altitude, signal to noise ratio (SNR), modulation error rate (MER), before correction bit-error rate (Pre BER) and after correction bit-error rates (Post BER) were measured and recorded at twenty (20) different locations in each zone ranging between $1 \mathrm{~km}$ to $40 \mathrm{~km}$ radius distance from transmitting antenna using GPS, Yaggi receiving external antenna set at a fixed height of $10 \mathrm{~m}$ and connected with the signal analyzer.

\section{Results and Discussion}

Table 1 to 6 present the results from the signal strength (SS), altitude, signal to noise ratio (SNR), modulation error rate (MER), before correction bit-error rate (preBER) and after correction biterror rate (postBER) for the two transmitting frequencies (728 and $762 \mathrm{MHz}$ ) measured along the six route at different distance location up to $40 \mathrm{~km}$ distance from the Startimes television transmission site.

From the tables, the pre BER ranging from $10^{-2}$ to $10^{-4}$ obtained for the two frequencies is good enough to produce post BER correction of $10^{-8}$. The obtained BER is in agreement with the ITU recommendation (ITU_R.BT.1306, 2011) which state that the post BER correction must be at least $10^{-6}$. This implies that the post BER of Startimes is above the minimum required for a quality delivery of DTTV at the users end. The signal to noise ratio (SNR) which was between 28 to $32 \mathrm{db}$ obtained in the tables for the two frequencies falls within the ITU recommendation for a stable signal which was stated in (ITU_R.F.339-8, 2013). The modulation error rate (MER) of between 25 to $32 \mathrm{db}$ obtained in the tables falls within the ETSI recommendation for a 
Fountain Journal of Natural and Applied Sciences: 2018; 7(1): 1 - 11

Table 1. Signal data for the frequencies $738 \mathrm{~Hz}$ and $762 \mathrm{~Hz}$ along Oyo road, Ibadan

\begin{tabular}{|c|c|c|c|c|c|c|c|c|c|c|c|c|c|c|}
\hline \multirow[t]{2}{*}{$\begin{array}{l}\text { Test } \\
\text { point }\end{array}$} & \multicolumn{2}{|c|}{ GPS Coordinate } & \multirow{2}{*}{$\begin{array}{l}\text { Distance } \\
\text { from } \\
\text { transmitting } \\
\text { station }(\mathrm{km})\end{array}$} & \multirow[t]{2}{*}{$\begin{array}{l}\text { Altitude } \\
\text { (Ft) }\end{array}$} & \multicolumn{2}{|c|}{$\begin{array}{l}\text { Power level } \\
(\mathrm{dB} \mu \mathrm{V})\end{array}$} & \multicolumn{2}{|c|}{$\begin{array}{c}\text { SNR } \\
(\mathrm{dB} \mu \mathrm{m} / \mathrm{m})\end{array}$} & \multicolumn{2}{|c|}{ MER (dB) } & \multicolumn{2}{|l|}{ bBER } & \multicolumn{2}{|l|}{ aBER } \\
\hline & Longitude & Latitude & & & 738 & 762 & 738 & 762 & 738 & 762 & 738 & 762 & 738 & 762 \\
\hline 1 & $3^{\circ} 54^{\prime} 36^{\prime \prime} \mathrm{E}$ & $7^{\circ} 25^{\prime} 25^{\prime \prime} \mathrm{N}$ & 3.2 & 737 & 77.0 & 75.4 & 30 & 30 & 31.1 & 30.6 & $1 \times 10^{-4}$ & $2 \times 10^{-4}$ & $<10^{-8}$ & $<10^{-8}$ \\
\hline 2 & $3^{\circ} 54^{\prime} 30^{\prime \prime} \mathrm{E}$ & $7^{\circ} 25^{\prime} 53^{\prime \prime} \mathrm{N}$ & 4.2 & 730 & 74.7 & 72.5 & 30 & 31 & 30 & 30 & $1 \times 10^{-4}$ & $1 \times 10^{-4}$ & $<10^{-s}$ & $<10^{-8}$ \\
\hline 3 & $3^{\circ} 54^{\prime} 25^{\prime \prime} \mathrm{E}$ & $7^{\circ} 26^{\prime} 45^{\prime \prime} \mathrm{N}$ & 5.7 & 689 & 66.9 & 65.2 & 31 & 31 & 32 & 31.6 & $1 \times 10^{-4}$ & $1 \times 10^{-4}$ & $<10^{-8}$ & $<10^{-8}$ \\
\hline 4 & $3^{\circ} 54^{\prime} 45^{\prime \prime} \mathrm{E}$ & $7^{\circ} 26^{\prime} 58^{\prime \prime} \mathrm{N}$ & 6.5 & 700 & 70.3 & 70.1 & 31 & 31 & 32 & 32 & $1 \times 10^{-4}$ & $1 \times 10^{-4}$ & $<10^{-8}$ & $<10^{-8}$ \\
\hline 5 & $3^{\circ} 54^{\prime} 49^{\prime \prime} \mathrm{E}$ & $7^{\circ} 27^{\prime} 40^{\prime \prime} \mathrm{N}$ & 7.7 & 725 & 68.1 & 68.3 & 31 & 31 & 32 & 32 & $1 \times 10^{-4}$ & $1 \times 10^{-4}$ & $<10^{-8}$ & $<10^{-8}$ \\
\hline 6 & $3^{\circ} 54^{\prime} 51^{\prime \prime} \mathrm{E}$ & $7^{\circ} 28^{\prime} 34^{\prime \prime} \mathrm{N}$ & 8.9 & 746 & 65.3 & 65.0 & 31 & 31 & 29 & 31.5 & $6 \times 10^{-4}$ & $1 \times 10^{-4}$ & $<10^{-8}$ & $<10^{-8}$ \\
\hline 7 & $3^{\circ} 54^{\prime} 55^{\prime \prime} \mathrm{E}$ & $7^{\circ} 28^{\prime} 45^{\prime \prime} \mathrm{N}$ & 9.8 & 750 & 63.1 & 63.4 & 31 & 31 & 29 & 29 & $1 \times 10^{-4}$ & $1 \times 10^{-4}$ & $<10^{-8}$ & $<10^{-8}$ \\
\hline 8 & $3^{\circ} 54^{\prime} 58^{\prime \prime} \mathrm{E}$ & $7^{\circ} 29^{\prime} 52^{\prime \prime} \mathrm{N}$ & 11.3 & 763 & 65.5 & 64.1 & 31 & 31 & 32 & 32 & $1 \times 10^{-4}$ & $1 \times 10^{-4}$ & $<10^{-8}$ & $<10^{-8}$ \\
\hline 9 & $3^{\circ} 55^{\prime} 06^{\prime \prime} \mathrm{E}$ & $7^{\circ} 31^{\prime} 04^{\prime \prime} \mathrm{N}$ & 13.6 & 829 & 69.6 & 67.9 & 31 & 32 & 32 & 32 & $2 \times 10^{-4}$ & $1 \times 10^{-4}$ & $<10^{-8}$ & $<10^{-8}$ \\
\hline 10 & $3^{\circ} 54^{\prime} 57^{\prime \prime} \mathrm{E}$ & $7^{\circ} 32^{\prime} 26^{\prime \prime} \mathrm{N}$ & 16.1 & 786 & 59.4 & 58.2 & 31 & 31 & 32 & 32 & $1 \times 10^{-4}$ & $1 \times 10^{-4}$ & $<10-^{8}$ & $<10^{-8}$ \\
\hline 11 & $3^{\circ} 54^{\prime} 55^{\prime \prime} \mathrm{E}$ & $7^{\circ} 35^{\prime} 33^{\prime \prime} \mathrm{N}$ & 21.8 & 862 & 51.9 & 50.2 & 30 & 28 & 30.9 & 28.8 & $2 \times 10^{-4}$ & $4 \times 10^{-4}$ & $<10^{-s}$ & $<10^{-8}$ \\
\hline 12 & $3^{\circ} 55^{\prime} 06^{\prime \prime} \mathrm{E}$ & $7^{\circ} 36^{\prime} 42^{\prime \prime} \mathrm{N}$ & 24 & 916 & 48.3 & 47.5 & 25 & 24 & 25.5 & 24.6 & $2 \times 10^{-3}$ & $2 \times 10^{-3}$ & $<10^{-8}$ & $<10^{-8}$ \\
\hline 13 & $3^{\circ} 55^{\prime} 15^{\prime \prime} \mathrm{E}$ & $7^{\circ} 37^{\prime} 42^{\prime \prime} \mathrm{N}$ & 25.9 & 927 & 55.4 & 53.1 & 31 & 24.5 & 32 & 32 & $2 \times 10^{-4}$ & $2 \times 10^{-4}$ & $<10^{-8}$ & $<10^{-8}$ \\
\hline 14 & $3^{\circ} 55^{\prime} 12^{\prime \prime} \mathrm{E}$ & $7^{\circ} 39^{\prime} 17^{\prime} \mathrm{N}$ & 27.3 & 923 & 51.0 & 51.0 & 26 & 26 & 32 & 31 & $9 \times 10^{-4}$ & $1 \times 10^{-3}$ & $<10^{-8}$ & $<10^{-8}$ \\
\hline 15 & $3^{\circ} 54^{\prime} 59^{\prime \prime} \mathrm{E}$ & $7^{\circ} 42^{\prime} 21^{\prime \prime} \mathrm{N}$ & 28.1 & 1054 & 50.5 & 50.1 & 26 & 25 & 31 & 31 & $2 \times 10^{-4}$ & $2 \times 10^{-4}$ & $<10^{-8}$ & $<10^{-8}$ \\
\hline 16 & $3^{\circ} 54^{\prime} 58^{\prime \prime} \mathrm{E}$ & $7^{\circ} 42^{\prime} 31^{\prime \prime} \mathrm{N}$ & 30.5 & 1067 & 47.1 & 47.0 & 26 & 26 & 31 & 31 & $1 \mathrm{X} 10^{-2}$ & $1 \times 10^{-2}$ & $<10^{-s}$ & $<10^{-8}$ \\
\hline 17 & $3^{\circ} 55^{\prime} 08^{\prime \prime} \mathrm{E}$ & $7^{\circ} 45^{\prime} 41^{\prime \prime} \mathrm{N}$ & 33.1 & 962 & 45.0 & 44.2 & 28 & 28 & 31 & 31 & $2 \times 10^{-4}$ & $1 \times 10^{-2}$ & $<10^{-8}$ & $<10^{-8}$ \\
\hline 18 & $3^{\circ} 54^{\prime} 57^{\prime \prime} \mathrm{E}$ & $7^{\circ} 48^{\prime} 43^{\prime \prime} \mathrm{N}$ & 35.3 & 1014 & 43.7 & 43.1 & 28 & 28 & 31 & 31 & $1 \times 10^{-2}$ & $1 \times 10^{-2}$ & $<10^{-s}$ & $<10^{-8}$ \\
\hline 19 & $3^{\circ} 55^{\prime} 10^{\prime \prime} \mathrm{E}$ & $7^{\circ} 49^{\prime} 08^{\prime \prime} \mathrm{N}$ & 37.5 & 1008 & 40.0 & 39.3 & 28 & 28 & 31 & 31 & $1 \times 10^{-2}$ & $1 \times 10^{-2}$ & $<10^{-8}$ & $<10^{-8}$ \\
\hline 20 & $3^{\circ} 55^{\prime} 34^{\prime \prime} \mathrm{E}$ & $7^{\circ} 49^{\prime} 56^{\prime \prime} \mathrm{N}$ & 40.5 & 965 & 37.9 & 37.5 & 28 & 28 & 31 & 31 & $1 \times 10^{-2}$ & $1 \times 10^{-2}$ & $<10^{-s}$ & $<10^{-8}$ \\
\hline
\end{tabular}

Table 2. Signal data for the frequencies $738 \mathrm{~Hz}$ and $762 \mathrm{~Hz}$ along Akobo road, Ibadan

\begin{tabular}{|c|c|c|c|c|c|c|c|c|c|c|c|c|c|c|}
\hline \multirow[t]{2}{*}{$\begin{array}{l}\text { Test } \\
\text { point }\end{array}$} & \multicolumn{2}{|c|}{ GPS Coordinate } & \multirow{2}{*}{$\begin{array}{l}\text { Distance } \\
\text { from } \\
\text { transmitting } \\
\text { station }(\mathrm{km})\end{array}$} & \multirow[t]{2}{*}{$\begin{array}{l}\text { Altitude } \\
(\mathrm{Ft})\end{array}$} & \multicolumn{2}{|c|}{$\begin{array}{l}\text { Power level } \\
(\mathrm{dB} \mu N)\end{array}$} & \multicolumn{2}{|c|}{$\mathrm{SNR}(\mathrm{dB} \mu \mathrm{V} / \mathrm{m})$} & \multicolumn{2}{|c|}{ MER (dB) } & \multicolumn{2}{|l|}{ bBER } & \multicolumn{2}{|l|}{ aBER } \\
\hline & Longitude & Latitude & & & 738 & 762 & 738 & 762 & 738 & 762 & 738 & 762 & 738 & 762 \\
\hline 1 & $3^{\circ} 54^{\prime} 54^{\prime \prime} \mathrm{E}$ & $7^{0} 25^{\prime} 15^{\prime \prime} \mathrm{N}$ & 3.4 & 725 & 74,2 & 71.9 & 31 & 32 & 31,1 & 30.6 & $1 \times 10^{-4}$ & $2 \times 10^{-4}$ & $<10^{-8}$ & $<10^{-8}$ \\
\hline 2 & $3^{\circ} 54^{\prime} 30^{\prime \prime} \mathrm{E}$ & $7^{0} 25^{\prime} 20^{\prime \prime} \mathrm{N}$ & 4.3 & 720 & 72,3 & 71.8 & 31 & 32 & 30 & 30 & $1 \times 10^{-4}$ & $1 \times 10^{-4}$ & $<10^{-8}$ & $<10^{-8}$ \\
\hline 3 & $3^{\circ} 54^{\prime} 14 " \mathrm{E}$ & $7^{0} 25^{\prime} 25^{\prime \prime} \mathrm{N}$ & 5.7 & 707 & 66.2 & 65.2 & 32 & 32 & 32 & 31.6 & $1 \times 10^{-4}$ & $1 \times 10^{-4}$ & $<10^{-8}$ & $<10^{-8}$ \\
\hline 4 & $3^{\circ} 55^{\prime} 59^{\prime \prime} \mathrm{E}$ & $7^{\circ} 25^{\prime} 34^{\prime \prime} \mathrm{N}$ & 6,7 & 801 & 75.0 & 72.0 & 32 & 31 & 32 & 32 & $1 \times 10^{-4}$ & $1 \times 10^{-4}$ & $<10^{-8}$ & $<10^{-8}$ \\
\hline 5 & $3^{\circ} 56^{\prime} 22^{\prime \prime} \mathrm{E}$ & $7^{\circ} 25^{\prime} 44^{\prime \prime} \mathrm{N}$ & 7.7 & 794 & 71.0 & 70.0 & 31 & 30 & 32 & 32 & $1 \times 10^{-4}$ & $1 \times 10^{-4}$ & $<10^{-8}$ & $<10^{-8}$ \\
\hline 6 & $3^{\circ} 56^{\prime} 35^{\prime \prime} \mathrm{E}$ & $7^{0} 25^{\prime} 58^{\prime \prime} \mathrm{N}$ & 9 & 805 & 69.3 & 69.5 & 31 & 31 & 29 & 31.5 & $6 \times 10^{-4}$ & $1 \times 10^{-4}$ & $<10^{-8}$ & $<10^{-8}$ \\
\hline 7 & $3^{\circ} 56^{\prime} 51^{\prime \prime} \mathrm{E}$ & $7^{\circ} 26^{\prime} 14^{\prime \prime} \mathrm{N}$ & 9.9 & 813 & 77.6 & 76.5 & 31 & 31 & 29 & 29 & $1 \times 10^{-4}$ & $1 \times 10^{-4}$ & $<10^{-8}$ & $<10^{-8}$ \\
\hline 8 & $3^{\circ} 56^{\prime} 57^{\prime \prime} \mathrm{E}$ & $7^{0} 26^{\prime} 50^{\prime \prime} \mathrm{N}$ & 11,3 & 823 & 72.5 & 72.1 & 31 & 31 & 32 & 32 & $1 \times 10^{-4}$ & $1 \times 10^{-4}$ & $<10^{-8}$ & $<10^{-8}$ \\
\hline 9 & $3^{\circ} 57^{\prime} 10^{\prime \prime} \mathrm{E}$ & $7^{\circ} 27^{\prime} 30^{\prime \prime} \mathrm{N}$ & 13.5 & 816 & 71.8 & 70,2 & 32 & 32 & 32 & 32 & $2 \times 10^{-4}$ & $1 \times 10^{-4}$ & $<10^{-8}$ & $<10^{-6}$ \\
\hline 10 & $3^{\circ} 57^{\prime} 20^{\prime \prime} \mathrm{E}$ & $7^{\circ} 27^{\prime} 55^{\prime \prime} \mathrm{N}$ & 16 & 828 & 68.1 & 67.3 & 31 & 31 & 32 & 32 & $1 \times 10^{-4}$ & $1 \times 10^{-4}$ & $<10^{-8}$ & $<10^{-8}$ \\
\hline 11 & $3^{\circ} 57^{\prime} 28^{\prime \prime} \mathrm{E}$ & $7^{\circ} 28^{\prime} 23^{\prime \prime} \mathrm{N}$ & 21.5 & 837 & 65,1 & 64.8 & 31 & 31 & 30.9 & 28,8 & $2 \times 10^{-4}$ & $4 \times 10^{-4}$ & $<10^{-8}$ & $<10^{-8}$ \\
\hline 12 & $3^{\circ} 57^{\prime} 55^{\prime \prime} \mathrm{E}$ & $7^{\circ} 28^{\prime} 37^{\prime \prime} \mathrm{N}$ & 24,3 & 830 & 63.0 & 62.5 & 31 & 31 & 25.5 & 24.6 & $2 \times 10^{-3}$ & $2 \times 10^{-3}$ & $<10^{-8}$ & $<10^{-6}$ \\
\hline 13 & $3^{\circ} 58^{\prime} 14^{\prime \prime} \mathrm{E}$ & $7^{0} 28^{\prime} 55^{\prime \prime} \mathrm{N}$ & 26 & 795 & 60.5 & 60.0 & 32 & 32 & 32 & 32 & $2 \times 10^{-4}$ & $2 \times 10^{-4}$ & $<10^{-8}$ & $<10^{-8}$ \\
\hline 14 & $3^{\circ} 58^{\prime} 40^{\prime \prime} \mathrm{E}$ & $7^{\circ} 28^{\circ} 05^{\prime \prime} \mathrm{N}$ & 27,2 & 900 & 58.4 & 58.2 & 31 & 31 & 32 & 31 & $9 \times 10^{-4}$ & $1 \times 10^{-3}$ & $<10^{-8}$ & $<10^{-8}$ \\
\hline 15 & $3^{\circ} 59^{\prime} 21^{\prime \prime} \mathrm{E}$ & $7^{\circ} 29^{\prime} 24^{\prime \prime} \mathrm{N}$ & 28,3 & 736 & 55.5 & 54.5 & 31 & 30 & 31 & 31 & $2 \times 10^{-4}$ & $2 \times 10^{-4}$ & $<10^{-8}$ & $<10^{-6}$ \\
\hline 16 & $3^{\circ} 59^{\prime} 52^{\prime \prime} \mathrm{E}$ & $7^{0} 29^{\prime} 55^{\prime \prime} \mathrm{N}$ & 30,3 & 855 & 54,1 & 53.2 & 31 & 31 & 31 & 31 & $1 \times 10^{-2}$ & $1 \times 10^{-2}$ & $<10^{-8}$ & $<10^{-8}$ \\
\hline 17 & $4^{\circ} 00^{\prime} 08^{\prime \prime} \mathrm{E}$ & $7^{\circ} 30^{\prime} 04^{\prime \prime} \mathrm{N}$ & 33 & 714 & 48,1 & 47.5 & 32 & 31 & 31 & 31 & $2 \times 10^{-4}$ & $1 \times 10^{-2}$ & $<10^{-8}$ & $<10^{-8}$ \\
\hline 18 & $4^{\circ} 00^{\prime} 55^{\prime \prime} \mathrm{E}$ & $7^{\circ} 30^{\prime} 25^{\prime \prime} \mathrm{N}$ & 35.5 & 820 & 45,1 & 44.3 & 31 & 31 & 31 & 31 & $1 \times 10^{-2}$ & $1 \times 10^{-2}$ & $<10^{-8}$ & $<10^{-8}$ \\
\hline 19 & $4^{\circ} 01^{\prime} 10^{\prime \prime} \mathrm{E}$ & $7^{\circ} 30^{\prime} 51^{\prime \prime N}$ & 37 & 915 & 40,3 & 40,1 & 31 & 31 & 31 & 31 & $1 \times 10^{-2}$ & $1 \times 10^{-2}$ & $<10^{-8}$ & $<10^{-8}$ \\
\hline 20 & $4^{\circ} 01^{\prime} 50 " \mathrm{E}$ & $7^{\circ} 31^{\prime} 05^{\prime \prime} \mathrm{N}$ & 40 & 960 & 37.8 & 37.5 & 31 & 31 & 31 & 31 & $1 \times 10^{-2}$ & $1 \times 10^{-2}$ & $<10^{-8}$ & $<10^{-8}$ \\
\hline
\end{tabular}


Fountain Journal of Natural and Applied Sciences: 2018; 7(1): 1 - 11

Table 3. Signal data for the frequencies $738 \mathrm{~Hz}$ and $762 \mathrm{~Hz}$ along Iwo road, Ibadan

\begin{tabular}{|c|c|c|c|c|c|c|c|c|c|c|c|c|c|c|}
\hline \multirow[t]{2}{*}{$\begin{array}{l}\text { Test } \\
\text { point }\end{array}$} & \multicolumn{2}{|c|}{ GPS Coordinate } & \multirow{2}{*}{$\begin{array}{l}\text { Distance } \\
\text { from } \\
\text { transmitting } \\
\text { station }(\mathrm{km})\end{array}$} & \multirow[t]{2}{*}{$\begin{array}{l}\text { Altitude } \\
(\mathrm{Ft})\end{array}$} & \multicolumn{2}{|c|}{$\begin{array}{l}\text { Power level } \\
(\mathrm{dB} \mu N)\end{array}$} & \multicolumn{2}{|c|}{$\mathrm{SNR}(\mathrm{dB} \mu \mathrm{V} / \mathrm{m})$} & \multicolumn{2}{|c|}{ MER (dB) } & \multicolumn{2}{|l|}{ bBER } & \multicolumn{2}{|l|}{ aBER } \\
\hline & Longitude & Latitude & & & 738 & 762 & 738 & 762 & 738 & 762 & 738 & 762 & 738 & 762 \\
\hline 1 & $3^{\circ} 56^{\prime} 30^{\prime \prime} \mathrm{E}$ & $7^{\circ} 24^{\prime} 15^{\prime \prime} \mathrm{N}$ & 3.2 & 821 & 76,1 & 76.7 & 32 & 32 & 32 & 32 & $1 \times 10^{-4}$ & $1 \times 10^{-4}$ & $<10^{-8}$ & $<10^{-6}$ \\
\hline 2 & $3^{\circ} 56^{\prime} 58^{\prime \prime} \mathrm{E}$ & $7^{\circ} 24^{\prime} 51^{\prime \prime} \mathrm{N}$ & 4,5 & 835 & 74,3 & 74.1 & 31 & 31 & 32 & 32 & $1 \times 10^{-4}$ & $1 \times 10^{-4}$ & $<10^{-8}$ & $<10^{-8}$ \\
\hline 3 & $3^{\circ} 57^{\prime} 24^{\prime \prime} \mathrm{E}$ & $7^{\circ} 25^{\prime} 00^{\prime \prime} \mathrm{N}$ & 5.5 & 740 & 64.1 & 63.9 & 30 & 30 & 32 & 32 & $2 \times 10^{-3}$ & $2 \times 10^{-3}$ & $<10^{-8}$ & $<10^{-8}$ \\
\hline 4 & $3^{\circ} 57^{\prime} 50^{\prime \prime} \mathrm{E}$ & $7^{\circ} 25^{\prime} 00^{\prime \prime} \mathrm{N}$ & 6.5 & 755 & 64.0 & 63.6 & 30 & 30 & 31 & 31 & $1 \times 10^{-4}$ & $1 \times 10^{-4}$ & $<10^{-8}$ & $<10^{-8}$ \\
\hline 5 & $3^{\circ} 58^{\prime} 25^{\prime \prime} \mathrm{E}$ & $7^{\circ} 25^{\prime} 13^{\prime \prime} \mathrm{N}$ & 7.5 & 790 & 60,8 & 60.5 & 30 & 30 & 31 & 31 & $1 \times 10^{-4}$ & $1 \times 10^{-4}$ & $<10^{-8}$ & $<10^{-8}$ \\
\hline 6 & $3^{\circ} 59^{\prime} 09^{\prime \prime} \mathrm{E}$ & $7^{\circ} 25^{\prime} 27^{\prime \prime} \mathrm{N}$ & 8.1 & 644 & 58.3 & 58.1 & 29 & 29 & 32 & 32 & $1 \times 10^{-3}$ & $4 \times 10^{-8}$ & $<10^{-8}$ & $<10^{-8}$ \\
\hline 7 & $3^{\circ} 59^{\prime} 45^{\prime \prime} \mathrm{E}$ & $7^{\circ} 25^{\prime} 35^{\prime \prime} \mathrm{N}$ & 9.2 & 700 & 61,3 & 60.8 & 30 & 30 & 31 & 31 & $1 \times 10^{-4}$ & $1 \times 10^{-4}$ & $<10^{-8}$ & $<10^{-8}$ \\
\hline 8 & $4^{\circ} 00^{\prime} 14^{\prime \prime} \mathrm{E}$ & $7^{\circ} 25^{\prime} 50^{\prime \prime} \mathrm{N}$ & 10.3 & 657 & 66.4 & 66.4 & 32 & 31 & 32 & 32 & $1 \times 10^{-4}$ & $1 \times 10^{-4}$ & $<10^{-8}$ & $<10^{-8}$ \\
\hline 9 & $4^{\circ} 00^{\prime} 52^{\prime \prime} \mathrm{E}$ & $7^{\circ} 26^{\circ} 05^{\prime \prime} \mathrm{N}$ & 11,2 & 690 & 62,5 & 62.0 & 31 & 31 & 32 & 32 & $1 \times 10^{-4}$ & $1 \times 10^{-4}$ & $<10^{-8}$ & $<10^{-8}$ \\
\hline 10 & $4^{\circ} 01^{\prime} 00^{\prime \prime} \mathrm{E}$ & $7^{\circ} 26^{\circ} 40^{\prime \prime} \mathrm{N}$ & 12.2 & 615 & 58,4 & 58.0 & 32 & 32 & 32 & 32 & $1 \times 10^{-4}$ & $1 \times 10^{-4}$ & $<10^{-8}$ & $<10^{-8}$ \\
\hline 11 & $4^{\circ} 02^{\prime} 09^{\prime \prime} E$ & $7^{\circ} 27^{\prime} 05^{\prime \prime} \mathrm{N}$ & 14.4 & 705 & 71.5 & 71.4 & 32 & 32 & 32 & 32 & $1 \times 10^{-4}$ & $1 \times 10^{-4}$ & $<10^{-8}$ & $<10^{-8}$ \\
\hline 12 & $4^{\circ} 03^{\prime} 39^{\prime \prime} \mathrm{E}$ & $7^{\circ} 27^{\prime} 30^{\prime \prime} \mathrm{N}$ & 17,2 & 705 & 51.9 & 52.0 & 30 & 30 & 30 & 31 & $1 \times 10^{-4}$ & $1 \times 10^{-4}$ & $<10^{-8}$ & $<10^{-8}$ \\
\hline 13 & $4^{\circ} 04^{\prime} 25^{\prime \prime} \mathrm{E}$ & $7^{\circ} 28^{\prime} 15^{\prime \prime} \mathrm{N}$ & 19.1 & 655 & 50,1 & 50.0 & 30 & 30 & 31 & 31 & $1 \times 10^{-4}$ & $1 \times 10^{-4}$ & $<10^{-8}$ & $<10^{-8}$ \\
\hline 14 & $4^{\circ} 04^{\prime} 53^{\prime \prime} E$ & $7^{\circ} 29^{\prime} 58^{\prime \prime} \mathrm{N}$ & 21.4 & 640 & 48,3 & 48.1 & 30 & 30 & 31 & 32 & $1 \times 10^{-4}$ & $1 \times 10^{-4}$ & $<10^{-8}$ & $<10^{-8}$ \\
\hline 15 & $4^{\circ} 05^{\prime} 38^{\prime \prime} E$ & $7^{\circ} 32^{\prime} 12^{\prime \prime} \mathrm{N}$ & 25 & 671 & 46.7 & 46.5 & 30 & 30 & 31 & 31 & $1 \times 10^{-4}$ & $1 \times 10^{-4}$ & $<10^{-8}$ & $<10^{-8}$ \\
\hline 16 & $4^{\circ} 06^{\prime} 03^{\prime \prime} E$ & $7^{\circ} 33^{\prime} 25^{\prime \prime} \mathrm{N}$ & 27 & 667 & 45,8 & 45.0 & 30 & 30 & 31 & 31 & $1 \times 10^{-4}$ & $1 \times 10^{-4}$ & $<10^{-8}$ & $<10^{-8}$ \\
\hline 17 & $4^{\circ} 06^{\prime} 43^{\prime \prime} \mathrm{E}$ & $7^{\circ} 33^{\prime} 51^{\prime \prime} \mathrm{N}$ & 29.1 & 730 & 43.5 & 43.1 & 30 & 30 & 31 & 31 & $1 \times 10^{-4}$ & $1 \times 10^{-4}$ & $<10^{-8}$ & $<10^{-8}$ \\
\hline 18 & $4^{\circ} 07^{\prime} 01^{\prime \prime} \mathrm{E}$ & $7^{\circ} 33^{\prime} 09^{\prime \prime} \mathrm{N}$ & 31.5 & 750 & 40,7 & 40.2 & 30 & 30 & 31 & 31 & $1 \times 10^{-4}$ & $1 \times 10^{-4}$ & $<10^{-8}$ & $<10^{-8}$ \\
\hline 19 & $4^{\circ} 07^{\prime} 25^{\prime \prime} E$ & $7^{\circ} 33^{\prime} 34^{\prime \prime} \mathrm{N}$ & 35,1 & 780 & 38.9 & 38.3 & 30 & 30 & 31 & 31 & $1 \times 10^{-4}$ & $1 \times 10^{-4}$ & $<10^{-8}$ & $<10^{-6}$ \\
\hline
\end{tabular}

Table 4. Signal data for the frequencies $738 \mathrm{~Hz}$ and $762 \mathrm{~Hz}$ along Ife road, Ibadan

\begin{tabular}{|c|c|c|c|c|c|c|c|c|c|c|c|c|c|c|}
\hline \multirow[t]{2}{*}{$\begin{array}{l}\text { Test } \\
\text { point }\end{array}$} & \multicolumn{2}{|c|}{ GPS Coordinate } & \multirow{2}{*}{$\begin{array}{l}\text { Distance } \\
\text { from } \\
\text { transmitting } \\
\text { station }(\mathrm{km})\end{array}$} & \multirow[t]{2}{*}{$\begin{array}{l}\text { Altitude } \\
(\mathrm{Ft})\end{array}$} & \multicolumn{2}{|c|}{$\begin{array}{l}\text { Power level } \\
(\mathrm{dB} \mu \mathrm{V})\end{array}$} & \multicolumn{2}{|c|}{$S N R(d B \mu V / m)$} & \multicolumn{2}{|c|}{ MER (dB) } & \multicolumn{2}{|l|}{ bBER } & \multicolumn{2}{|l|}{ aBER } \\
\hline & Longitude & Latitude & & & 738 & 762 & 738 & 762 & 738 & 762 & 738 & 762 & 738 & 762 \\
\hline 1 & $3^{\circ} 06^{\prime} 55^{\prime \prime} \mathrm{E}$ & $7^{\circ} 23^{\prime} 57^{\prime \prime} \mathrm{N}$ & 3.5 & 782 & 75,1 & 74.3 & 31 & 32 & 31 & 32 & $9 \times 10^{-4}$ & $1 \times 10^{-4}$ & $<10^{-8}$ & $<10^{-8}$ \\
\hline 2 & $3^{\circ} 57^{\prime} 05^{\prime \prime} \mathrm{E}$ & $7^{\circ} 23^{\prime} 41^{\prime \prime} \mathrm{N}$ & 4.5 & 780 & 73.5 & 73.1 & 31 & 31 & 32 & 32 & $1 \times 10^{-4}$ & $1 \times 10^{-4}$ & $<10^{-8}$ & $<10^{-8}$ \\
\hline 3 & $3^{\circ} 57^{\prime} 57^{\prime \prime} \mathrm{E}$ & $7^{\circ} 23^{\prime} 37^{\prime \prime} \mathrm{N}$ & 5.3 & 791 & 71,3 & 71.3 & 31 & 30 & 32 & 32 & $2 \times 10^{-4}$ & $2 \times 10^{-3}$ & $<10^{-8}$ & $<10^{-8}$ \\
\hline 4 & $3^{\circ} 58^{\prime} 10^{\prime \prime} \mathrm{E}$ & $7^{0} 23^{\prime} 19^{\prime \prime} \mathrm{N}$ & 7 & 800 & 70.5 & 70.3 & 31 & 30 & 32 & 31 & $1 \times 10^{-4}$ & $1 \times 10^{-4}$ & $<10^{-8}$ & $<10^{-8}$ \\
\hline 5 & $3^{\circ} 58^{\prime} 55^{\prime \prime} \mathrm{E}$ & $7^{\circ} 23^{\prime} 02^{\prime \prime} \mathrm{N}$ & 8.5 & 805 & 68.3 & 68.5 & 31 & 30 & 32 & 31 & $1 \times 10^{-4}$ & $1 \times 10^{-4}$ & $<10^{-8}$ & $<10^{-8}$ \\
\hline 6 & $4^{\circ} 00^{\prime} 50^{\prime \prime} \mathrm{E}$ & $7^{\circ} 22^{\prime} 57^{\prime \prime} \mathrm{N}$ & 10,7 & 542 & 58.5 & 55.0 & 31 & 29 & 31 & 32 & $1 \times 10^{-4}$ & $4 \times 10^{-8}$ & $<10^{-8}$ & $<10^{-8}$ \\
\hline 7 & $4^{\circ} 01^{\prime} 56 " \mathrm{E}$ & $7^{\circ} 22^{\prime} 35^{\prime \prime} \mathrm{N}$ & 12,8 & 613 & 61.7 & 64.2 & 31 & 30 & 31 & 31 & $1 \times 10^{-4}$ & $1 \times 10^{-4}$ & $<10^{-8}$ & $<10^{-8}$ \\
\hline 8 & $4^{\circ} 04^{\prime} 29^{\prime \prime} \mathrm{E}$ & $7^{\circ} 22^{\prime} 22^{\prime \prime} \mathrm{N}$ & 17,6 & 644 & 62.5 & 66.5 & 31 & 31 & 32 & 32 & $6 \times 10^{-4}$ & $1 \times 10^{-4}$ & $<10^{-8}$ & $<10^{-8}$ \\
\hline 9 & $4^{\circ} 04^{\prime} 52^{\prime \prime} \mathrm{E}$ & $7^{\circ} 22^{\circ} 09^{\prime \prime} \mathrm{N}$ & 18,8 & 750 & 67,8 & 67.3 & 31 & 31 & 32 & 32 & $1 \times 10^{-4}$ & $1 \times 10^{-4}$ & $<10^{-8}$ & $<10^{-8}$ \\
\hline 10 & $4^{\circ} 05^{\prime} 54^{\prime \prime} \mathrm{E}$ & $7^{\circ} 22^{\prime} 01^{\prime \prime} \mathrm{N}$ & 20.1 & 755 & 57,4 & 56.7 & 32 & 32 & 32 & 32 & $1 \times 10^{-4}$ & $1 \times 10^{-4}$ & $<10^{-8}$ & $<10^{-8}$ \\
\hline 11 & $4^{\circ} 07^{\prime} 27^{\prime \prime} \mathrm{E}$ & $7^{\circ} 21^{\prime} 35^{\prime \prime} \mathrm{N}$ & 23.2 & 801 & 55.3 & 55.0 & 31 & 32 & 32 & 32 & $1 \times 10^{-2}$ & $1 \times 10^{-4}$ & $<10^{-8}$ & $<10^{-8}$ \\
\hline 12 & $4^{\circ} 08^{\prime} 31^{\prime \prime} \mathrm{E}$ & $7^{\circ} 21^{\prime} 15^{\prime \prime} \mathrm{N}$ & 25,2 & 785 & 52,8 & 52.5 & 31 & 30 & 32 & 31 & $9 \times 10^{-3}$ & $1 \times 10^{-3}$ & $<10^{-8}$ & $<10^{-8}$ \\
\hline 13 & $4^{\circ} 09^{\prime} 40^{\prime \prime} \mathrm{E}$ & $7^{\circ} 21^{\prime} 46^{\prime \prime} \mathrm{N}$ & 27,2 & 825 & 50.5 & 50.0 & 31 & 30 & 32 & 31 & $1 \times 10^{-2}$ & $1 \times 10^{-4}$ & $<10^{-8}$ & $<10^{-8}$ \\
\hline 14 & $4^{\circ} 10^{\prime} 41^{\prime \prime} \mathrm{E}$ & $7^{\circ} 21^{\prime} 35^{\prime \prime} \mathrm{N}$ & 28,8 & 830 & 52,0 & 51.5 & 31 & 30 & 32 & 32 & $1 \times 10^{-4}$ & $1 \times 10^{-4}$ & $<10^{-8}$ & $<10^{-8}$ \\
\hline 15 & $4^{\circ} 10^{\prime} 53^{\prime \prime} \mathrm{E}$ & $7^{\circ} 21^{\prime} 23^{\prime \prime} \mathrm{N}$ & 30,1 & 700 & 49.5 & 49.2 & 31 & 30 & 32 & 31 & $1 \times 10^{-4}$ & $1 \times 10^{-4}$ & $<10^{-8}$ & $<10^{-8}$ \\
\hline 16 & $4^{\circ} 11^{\prime} 07^{\prime \prime} \mathrm{E}$ & $7^{\circ} 21^{\prime} 04^{\prime \prime} \mathrm{N}$ & 32,5 & 713 & 48.8 & 48.5 & 31 & 30 & 32 & 31 & $1 \times 10^{-4}$ & $1 \times 10^{-4}$ & $<10^{-8}$ & $<10^{-8}$ \\
\hline 17 & $4^{\circ} 11^{\prime} 41^{\prime \prime E}$ & $7^{\circ} 20^{\prime} 53^{\prime \prime} \mathrm{N}$ & 35 & 900 & 46.9 & 46.3 & 31 & 30 & 32 & 31 & $1 \times 10^{-4}$ & $1 \times 10^{-4}$ & $<10^{-8}$ & $<10^{-8}$ \\
\hline 18 & $4^{\circ} 11^{\prime} 58^{\prime \prime} E$ & $7^{\circ} 20^{\prime} 40^{\prime \prime} \mathrm{N}$ & 37,5 & 905 & 43.5 & 42,5 & 31 & 30 & 32 & 31 & $1 \times 10^{-4}$ & $1 \times 10^{-4}$ & $<10^{-8}$ & $<10^{-8}$ \\
\hline 19 & $4^{\circ} 12^{\prime} 10^{\prime \prime} \mathrm{E}$ & $7^{\circ} 20^{\prime} 31^{\prime \prime} \mathrm{N}$ & 38.9 & 950 & 40,1 & 40.0 & 31 & 30 & 32 & 31 & $1 \times 10^{-4}$ & $1 \times 10^{-4}$ & $<10^{-8}$ & $<10^{-8}$ \\
\hline 20 & $4^{\circ} 12^{\prime} 38^{\prime \prime} \mathrm{E}$ & $7^{\circ} 20^{\prime} 14^{\prime \prime} \mathrm{N}$ & 40,1 & 961 & 40.0 & 38.5 & 31 & 30 & 32 & 31 & $1 \times 10^{-4}$ & $1 \times 10^{-4}$ & $<10^{-8}$ & $<10^{-8}$ \\
\hline
\end{tabular}


Fountain Journal of Natural and Applied Sciences: 2018; 7(1): 1 - 11

Table 5. Signal data for the frequencies $738 \mathrm{~Hz}$ and $762 \mathrm{~Hz}$ along Airport road, Ibadan

\begin{tabular}{|c|c|c|c|c|c|c|c|c|c|c|c|c|c|c|}
\hline \multirow[t]{2}{*}{$\begin{array}{l}\text { Test } \\
\text { point }\end{array}$} & \multicolumn{2}{|c|}{ GPS Coordinate } & \multirow{2}{*}{$\begin{array}{l}\text { Distance } \\
\text { from } \\
\text { transmitting } \\
\text { station }(\mathrm{km})\end{array}$} & \multirow[t]{2}{*}{$\begin{array}{l}\text { Altitude } \\
(\mathrm{Ft})\end{array}$} & \multicolumn{2}{|c|}{$\begin{array}{l}\text { Power level } \\
(\mathrm{dB} \mu V)\end{array}$} & \multicolumn{2}{|c|}{$S N R(d B \mu V / m)$} & \multicolumn{2}{|c|}{ MER (dB) } & \multicolumn{2}{|l|}{ bBER } & \multicolumn{2}{|l|}{ aBER } \\
\hline & Longitude & Latitude & & & 738 & 762 & 738 & 762 & 738 & 762 & 738 & 762 & 738 & 762 \\
\hline 1 & $3^{\circ} 53^{\prime} 56^{\prime \prime} \mathrm{E}$ & $7^{\circ} 22^{\prime} 47^{\prime \prime N} \mathrm{~N}$ & 2.7 & 813 & 73,1 & 71.2 & 32 & 32 & 32 & 32 & $1 \times 10^{-4}$ & $1 \times 10^{-4}$ & $<10^{-8}$ & $<10^{-8}$ \\
\hline 2 & $3^{\circ} 53^{\prime} 30^{\prime \prime} \mathrm{E}$ & $7^{\circ} 22^{\prime} 38^{\prime \prime} \mathrm{N}$ & 3.5 & 720 & 62.0 & 62.0 & 31 & 31 & 32 & 32 & $1 \times 10^{-4}$ & $1 \times 10^{-4}$ & $<10^{-8}$ & $<10^{-8}$ \\
\hline 3 & $3^{\circ} 53^{\prime} 31^{\prime \prime} \mathrm{E}$ & $7^{\circ} 21^{\prime} 43^{\prime \prime} \mathrm{N}$ & 4.7 & 728 & 65.9 & 64.5 & 30 & 30 & 32 & 32 & $2 \times 10^{-3}$ & $2 \times 10^{-3}$ & $<10^{-8}$ & $<10^{-8}$ \\
\hline 4 & $3^{\circ} 52^{\prime} 44^{\prime \prime} \mathrm{E}$ & $7^{\circ} 20^{\prime} 56^{\prime \prime} \mathrm{N}$ & 6.8 & 624 & 58,7 & 60.5 & 30 & 30 & 31 & 31 & $1 \times 10^{-4}$ & $1 \times 10^{-4}$ & $<10^{-8}$ & $<10^{-8}$ \\
\hline 5 & $3^{\circ} 52^{\prime} 20^{\prime \prime} \mathrm{E}$ & $7^{\circ} 20^{\prime} 31^{\prime \prime} \mathrm{N}$ & 7,8 & 794 & 80,7 & 80.2 & 30 & 30 & 31 & 31 & $1 \times 10^{-4}$ & $1 \times 10^{-4}$ & $<10^{-8}$ & $<10^{-8}$ \\
\hline 6 & $3^{\circ} 52^{\prime} 04^{\prime \prime} \mathrm{E}$ & $7^{\circ} 19^{\prime} 44^{\prime \prime} \mathrm{N}$ & 9.3 & 750 & 67.4 & 64.4 & 29 & 29 & 32 & 32 & $1 \times 10^{-3}$ & $4 \times 10^{-8}$ & $<10^{-8}$ & $<10^{-8}$ \\
\hline 7 & $3^{\circ} 52^{\prime} 10^{\prime \prime} \mathrm{E}$ & $7^{\circ} 20^{\prime} 23^{\prime \prime} \mathrm{N}$ & 10.5 & 813 & 70.0 & 70.3 & 30 & 30 & 31 & 31 & $1 \times 10^{-4}$ & $1 \times 10^{-4}$ & $<10^{-8}$ & $<10^{-8}$ \\
\hline 8 & $3^{\circ} 50^{\prime} 56 " \mathrm{E}$ & $7^{\circ} 20^{\prime} 10^{\prime \prime} \mathrm{N}$ & 11,7 & 823 & 78.5 & 78.6 & 32 & 31 & 32 & 32 & $1 \times 10^{-4}$ & $1 \times 10^{-4}$ & $<10^{-8}$ & $<10^{-8}$ \\
\hline 9 & $3^{\circ} 49^{\prime} 43^{\prime \prime} \mathrm{E}$ & $7^{\circ} 19^{\prime} 58^{\prime \prime} \mathrm{N}$ & 13,8 & 816 & 61,2 & 60.3 & 31 & 31 & 32 & 32 & $1 \times 10^{-4}$ & $1 \times 10^{-4}$ & $<10^{-8}$ & $<10^{-8}$ \\
\hline 10 & $3^{\circ} 50^{\prime} 24^{\prime \prime} \mathrm{E}$ & $7^{\circ} 21^{\prime} 03^{\prime \prime} \mathrm{N}$ & 14,5 & 828 & 74,6 & 73.1 & 32 & 32 & 32 & 32 & $1 \times 10^{-4}$ & $1 \times 10^{-4}$ & $<10^{-8}$ & $<10^{-8}$ \\
\hline 11 & $3^{\circ} 48^{\prime} 53^{\prime \prime} \mathrm{E}$ & $7^{\circ} 21^{\prime} 56^{\prime \prime} \mathrm{N}$ & 15.6 & 837 & 74,4 & 72.0 & 32 & 32 & 32 & 32 & $1 \times 10^{-3}$ & $1 \times 10^{-3}$ & $<10^{-8}$ & $<10^{-8}$ \\
\hline 12 & $3^{\circ} 49^{\prime} 00^{\prime \prime} \mathrm{E}$ & $7^{\circ} 22^{\prime} 34^{\prime \prime} \mathrm{N}$ & 16,5 & 790 & 66.3 & 66.3 & 30 & 30 & 30 & 31 & $1 \times 10^{-4}$ & $1 \times 10^{-4}$ & $<10^{-8}$ & $<10^{-8}$ \\
\hline 13 & $3^{\circ} 49^{\prime} 25^{\prime \prime} \mathrm{E}$ & $7^{\circ} 23^{\prime} 12^{\prime \prime} \mathrm{N}$ & 18 & 795 & 60,5 & 60.0 & 30 & 30 & 31 & 31 & $1 \times 10^{-4}$ & $1 \times 10^{-4}$ & $<10^{-8}$ & $<10^{-8}$ \\
\hline 14 & $3^{\circ} 48^{\prime} 02^{\prime \prime} \mathrm{E}$ & $7^{\circ} 23^{\prime} 54^{\prime \prime} \mathrm{N}$ & 20,3 & 750 & 58.7 & 58.0 & 30 & 30 & 31 & 32 & $1 \times 10^{-4}$ & $1 \times 10^{-4}$ & $<10^{-8}$ & $<10^{-8}$ \\
\hline 15 & $3^{\circ} 47^{\prime} 07^{\prime \prime} \mathrm{E}$ & $7^{\circ} 23^{\prime} 45^{\prime \prime} \mathrm{N}$ & 22.5 & 736 & 50,2 & 49.0 & 30 & 30 & 31 & 31 & $1 \times 10^{-4}$ & $1 \times 10^{-4}$ & $<10^{-8}$ & $<10^{-8}$ \\
\hline 16 & $3^{\circ} 23^{\prime} 58^{\prime \prime} \mathrm{E}$ & $7^{\circ} 46^{\circ} 02^{\prime \prime} \mathrm{N}$ & 25.3 & 730 & 48,5 & 48.0 & 30 & 30 & 31 & 31 & $1 \times 10^{-4}$ & $1 \times 10^{-4}$ & $<10^{-8}$ & $<10^{-8}$ \\
\hline 17 & $3^{\circ} 23^{\prime} 36^{\prime \prime} \mathrm{E}$ & $7^{\circ} 45^{\prime} 01^{\prime \prime} \mathrm{N}$ & 30.5 & 733 & 46,0 & 46.1 & 30 & 30 & 31 & 31 & $1 \times 10^{-4}$ & $1 \times 10^{-4}$ & $<10^{-8}$ & $<10^{-8}$ \\
\hline 18 & $3^{\circ} 44^{\circ} 46^{\prime \prime} \mathrm{E}$ & $7^{\circ} 23^{\prime} 20^{\prime \prime} \mathrm{N}$ & 33,1 & 700 & 45,8 & 45.3 & 30 & 30 & 31 & 31 & $1 \times 10^{-4}$ & $1 \times 10^{-4}$ & $<10^{-8}$ & $<10^{-8}$ \\
\hline 19 & $3^{\circ} 43^{\prime} 18^{\prime \prime} \mathrm{E}$ & $7^{\circ} 23^{\prime} 12^{\prime \prime} \mathrm{N}$ & 37,3 & 715 & 41.0 & 40.5 & 30 & 30 & 31 & 31 & $1 \times 10^{-4}$ & $1 \times 10^{-4}$ & $<10^{-8}$ & $<10^{-8}$ \\
\hline 20 & $3^{\circ} 42^{\prime} 02^{\prime \prime} \mathrm{E}$ & $7^{\circ} 23^{\prime} 43^{\prime \prime} \mathrm{N}$ & 40,3 & 703 & 37,1 & 36.9 & 30 & 30 & 31 & 31 & $1 \times 10^{-4}$ & $1 \times 10^{-4}$ & $<10^{-8}$ & $<10^{-8}$ \\
\hline
\end{tabular}

Table 6. Signal data for the frequencies $738 \mathrm{~Hz}$ and $762 \mathrm{~Hz}$ along Southwest road, Ibadan

\begin{tabular}{|c|c|c|c|c|c|c|c|c|c|c|c|c|c|c|}
\hline \multirow[t]{2}{*}{$\begin{array}{l}\text { Test } \\
\text { point }\end{array}$} & \multicolumn{2}{|c|}{ GPS Coordinate } & \multirow{2}{*}{$\begin{array}{l}\text { Distance } \\
\text { from } \\
\text { transmitting } \\
\text { station }(\mathrm{km})\end{array}$} & \multirow[t]{2}{*}{$\begin{array}{l}\text { Altitude } \\
(\mathrm{Ft})\end{array}$} & \multicolumn{2}{|c|}{$\begin{array}{l}\text { Power level } \\
(\mathrm{dB} \mu \mathrm{V})\end{array}$} & \multicolumn{2}{|c|}{$\mathrm{SNR}(\mathrm{dB} \mu \mathrm{V} / \mathrm{m})$} & \multicolumn{2}{|c|}{ MER (dB) } & \multicolumn{2}{|l|}{ bBER } & \multicolumn{2}{|l|}{ aBER } \\
\hline & Longitude & Latitude & & & 738 & 762 & 738 & 762 & 738 & 762 & 738 & 762 & 738 & 762 \\
\hline 1 & $3^{\circ 56}$ '56"E & $7^{\circ} 20^{\prime} 53^{\prime \prime} \mathrm{N}$ & 3.5 & 821 & 80.0 & 79.1 & 31 & 31 & 31 & 32 & $9 \times 10^{-4}$ & $1 \times 10^{-4}$ & $<10^{-8}$ & $<10^{-8}$ \\
\hline 2 & $3^{\circ} 57^{\prime} 10^{\prime \prime} \mathrm{E}$ & $7^{\circ} 20^{\prime} 40^{\prime \prime} \mathrm{N}$ & 5,5 & 835 & 75,1 & 74.1 & 31 & 31 & 32 & 32 & $1 \times 10^{-4}$ & $1 \times 10^{-4}$ & $<10^{-8}$ & $<10^{-8}$ \\
\hline 3 & 3057' 24"E & $7^{\circ} 20^{\prime} 34^{\prime \prime} \mathrm{N}$ & 6.8 & 740 & 73,3 & 73.2 & 31 & 30 & 32 & 32 & $2 \times 10^{-4}$ & $1 \times 10^{-4}$ & $<10^{-8}$ & $<10^{-8}$ \\
\hline 4 & $3^{\circ} 57^{\prime} 55^{\prime \prime} \mathrm{E}$ & $7^{\circ} 20^{\prime} 15^{\prime \prime} \mathrm{N}$ & 8.5 & 755 & 70,3 & 69.8 & 31 & 31 & 32 & 32 & $1 \times 10^{-4}$ & $1 \times 10^{-4}$ & $<10^{-8}$ & $<10^{-8}$ \\
\hline 5 & $3^{\circ} 58^{\prime} 21^{\prime \prime} \mathrm{E}$ & $7^{\circ} 20^{\prime} 02^{\prime \prime} \mathrm{N}$ & 9.2 & 790 & 72,3 & 72.0 & 31 & 31 & 32 & 32 & $1 \times 10^{-4}$ & $1 \times 10^{-4}$ & $<10^{-8}$ & $<10^{-8}$ \\
\hline 6 & $3^{\circ} 59^{\prime} 28^{\prime \prime} \mathrm{E}$ & $7^{\circ} 19^{\prime} 15^{\prime \prime} \mathrm{N}$ & 11,6 & 644 & 70.6 & 70.0 & 31 & 32 & 31 & 32 & $1 \times 10^{-4}$ & $1 \times 10^{-4}$ & $<10^{-8}$ & $<10^{-8}$ \\
\hline 7 & $4^{\circ} 00^{\prime} 41^{\prime \prime} \mathrm{E}$ & $7^{\circ} 18^{\prime} 30^{\prime \prime} \mathrm{N}$ & 14,2 & 700 & 69.0 & 70.6 & 31 & 30,1 & 31 & 30.8 & $1 \times 10^{-4}$ & $1 \times 10^{-4}$ & $<10^{-8}$ & $<10^{-8}$ \\
\hline 8 & $4^{\circ} 00^{\prime} 35^{\prime \prime} \mathrm{E}$ & $7^{\circ} 18^{\prime} 50^{\prime \prime} \mathrm{N}$ & 16 & 657 & 68.1 & 67.3 & 31 & 30 & 32 & 31.6 & $6 \times 10^{-4}$ & $1 \times 10^{-4}$ & $<10^{-8}$ & $<10^{-8}$ \\
\hline 9 & $4^{\circ} 01^{\prime} 30^{\prime \prime} \mathrm{E}$ & $7^{\circ} 17^{\prime} 05^{\prime \prime} \mathrm{N}$ & 17,1 & 690 & 65.8 & 62.3 & 31 & 27 & 32 & 32 & $1 \times 10^{-4}$ & $1 \times 10^{-4}$ & $<10^{-8}$ & $<10^{-8}$ \\
\hline 10 & $4^{\circ} 01^{\prime} 34^{\prime \prime} \mathrm{E}$ & $7^{\circ} 16^{\prime} 30^{\prime \prime} \mathrm{N}$ & 19 & 615 & 62.6 & 59.3 & 32 & 31 & 32 & 32 & $1 \times 10^{-4}$ & $1 \times 10^{-2}$ & $<10^{-8}$ & $<10^{-8}$ \\
\hline 11 & $4^{\circ} 01^{\prime} 43^{\prime \prime E}$ & $7^{\circ} 15,02 " \mathrm{~N}$ & 20,3 & 705 & 60,1 & 57.3 & 31 & 31 & 32 & 32 & $1 \times 10^{-2}$ & $1 \times 10^{-2}$ & $<10^{-8}$ & $<10^{-8}$ \\
\hline 12 & $4^{\circ} 01^{\prime} 36^{\prime \prime E}$ & $7^{\circ} 13^{\prime} 43^{\prime \prime N}$ & 22,2 & 705 & 57.0 & 54.1 & 31 & 31 & 32 & 32 & $9 \times 10^{-3}$ & $6 \times 10^{-3}$ & $<10^{-8}$ & $<10^{-8}$ \\
\hline 13 & $4^{\circ} 01^{\prime} 45^{\prime \prime} \mathrm{E}$ & $7^{\circ} 12^{\prime} 01^{\prime \prime} \mathrm{N}$ & 25,2 & 655 & 54,5 & 52.4 & 31 & 31 & 32 & 32 & $1 \times 10^{-2}$ & $1 \times 10^{-4}$ & $<10^{-8}$ & $<10^{-8}$ \\
\hline 14 & $4^{\circ} 01^{\prime} 59 " \mathrm{E}$ & $7^{\circ} 11^{\prime} 25^{\prime \prime} \mathrm{N}$ & 26,5 & 640 & 52,8 & 50.1 & 31 & 31 & 32 & 32 & $1 \times 10^{-4}$ & $3 \times 10^{-4}$ & $<10^{-8}$ & $<10^{-8}$ \\
\hline 15 & $4^{\circ} 02^{\prime} 02^{\prime \prime} \mathrm{E}$ & $7^{\circ} 10^{\prime} 24^{\prime \prime} \mathrm{N}$ & 27.9 & 671 & 50.5 & 48.3 & 31 & 31 & 32 & 32 & $1 \times 10^{-4}$ & $1 \times 10^{-4}$ & $<10^{-8}$ & $<10^{-8}$ \\
\hline 16 & $4002^{\prime} 50 " \mathrm{E}$ & $7^{\circ} 09^{\prime} 30^{\prime \prime} \mathrm{N}$ & 29,1 & 667 & 48,1 & 45.0 & 31 & 31 & 32 & 32 & $1 \times 10^{-4}$ & $2 \times 10^{-4}$ & $<10^{-8}$ & $<10^{-8}$ \\
\hline 17 & $4^{\circ} 01^{\prime} 44^{\prime \prime} \mathrm{E}$ & $7^{\circ} 07^{\prime} 45^{\prime \prime} \mathrm{N}$ & 31,1 & 730 & 45.7 & 42.5 & 31 & 31 & 32 & 32 & $1 \times 10^{-4}$ & $1 \times 10^{-4}$ & $<10^{-8}$ & $<10^{-8}$ \\
\hline 18 & $4^{\circ} 01^{\prime} 51^{\prime \prime} \mathrm{E}$ & $7^{\circ} 06^{\prime} 50^{\prime \prime} \mathrm{N}$ & 33.5 & 750 & 42.0 & 40.5 & 31 & 31 & 32 & 32 & $1 \times 10^{-4}$ & $1 \times 10^{-4}$ & $<10^{-8}$ & $<10^{-8}$ \\
\hline 19 & $4^{\circ} 01^{\prime} 57^{\prime \prime E}$ & $7^{\circ} 05^{\prime} 55^{\prime \prime} \mathrm{N}$ & 36,3 & 780 & 40,7 & 38.1 & 31 & 31 & 32 & 32 & $1 \times 10^{-4}$ & $1 \times 10^{-4}$ & $<10^{-8}$ & $<10^{-8}$ \\
\hline 20 & $4^{\circ} 02^{\prime} 20^{\prime \prime} \mathrm{E}$ & $7^{\circ} 05^{\prime} 10^{\prime \prime} \mathrm{N}$ & 40 & 801 & 38.0 & 37.0 & 31 & 31 & 32 & 32 & $1 \times 10^{-4}$ & $1 \times 10^{-4}$ & $<10^{-8}$ & $<10^{-8}$ \\
\hline
\end{tabular}


Quadrature Phase Shift keying (QPSK) modulation techniques employed by Startimes. This specification as stated in (ETSI TR 101290, 2001) recommended a minimum acceptable operational value of $18 \mathrm{~dB}$. Both the SNR and MER are quantities measured at the baseband. The $S N R$ is measured before modulation and this value have a significant effect on the MER, which is a measure of the quality of digitally modulated signal and it provides the figure of merit analysis of the signal in the assessment of the performance of a DVB-T system (Fortin, 2008). With the MER of Startimes above the minimum, this implies the performance of Startimes DVB$T$ system is good enough to deliver a High definition image.

The plot of the signal strength against distance presented in Figures 3 and 4 show some spikes in signal strength as it decrease with distance, these spikes are attributed to the effect of the altitude on the signal strength. This relationship can be vividly seen on the 3-D graph plotted in Figures 5 and 6 for the two frequencies. This finding is also correlated with the statistical analysis carried out using SPSS IBM presented in Tables 7 and 8 , which reveals the effect of altitude on the signal strength. From the correlation analysis in Table 7, there exists a positive relationship between the power level and altitude for the two transmitting frequencies. The correlation coefficients for the $728 \mathrm{MHz}$ and $738 \mathrm{MHz}$ are given to be $r=$ 0.618 and $r=0.510$ respectively. This relationship is insignificant considering their $p$ values of 0.057 and 0.132 respectively as compared to the test p-value of 0.05 . A negative relationship between the power level and distance was established with and $r$-value of 0.873 and $r$-value of 0.790 respectively. This relationship is significant at 0.001 and 0.007 respectively compared to the $P$-value of 0.05 .

A Paired T-test presented in Table 8 was further used to determine the effect of power level on the altitude and distance. The result showed that altitude affects the power level since

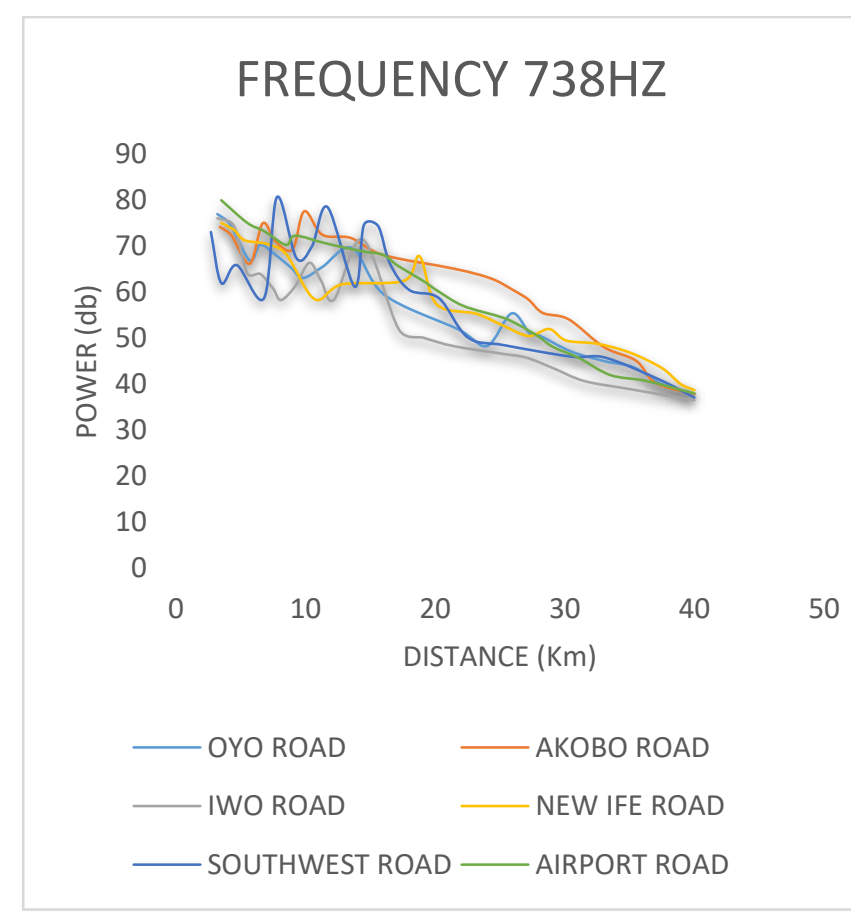

Figure 3. Plot of measured power against distance for frequency $738 \mathrm{~Hz}$

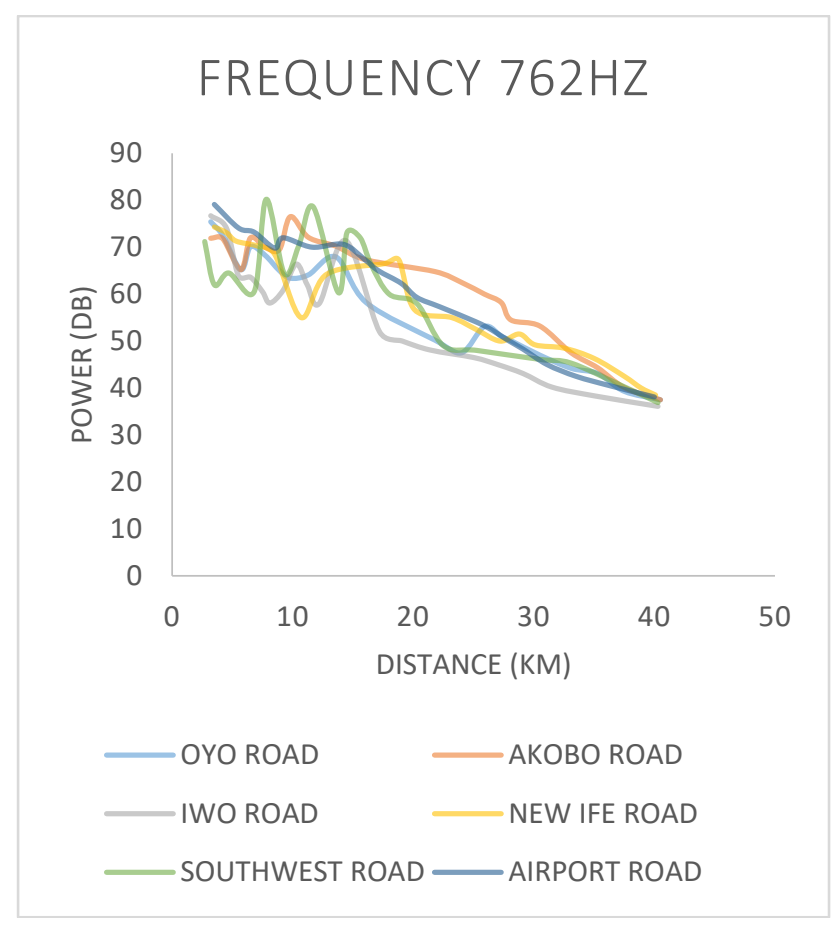

Figure 4. Plot of measured power against distance for frequency $762 \mathrm{~Hz}$ 


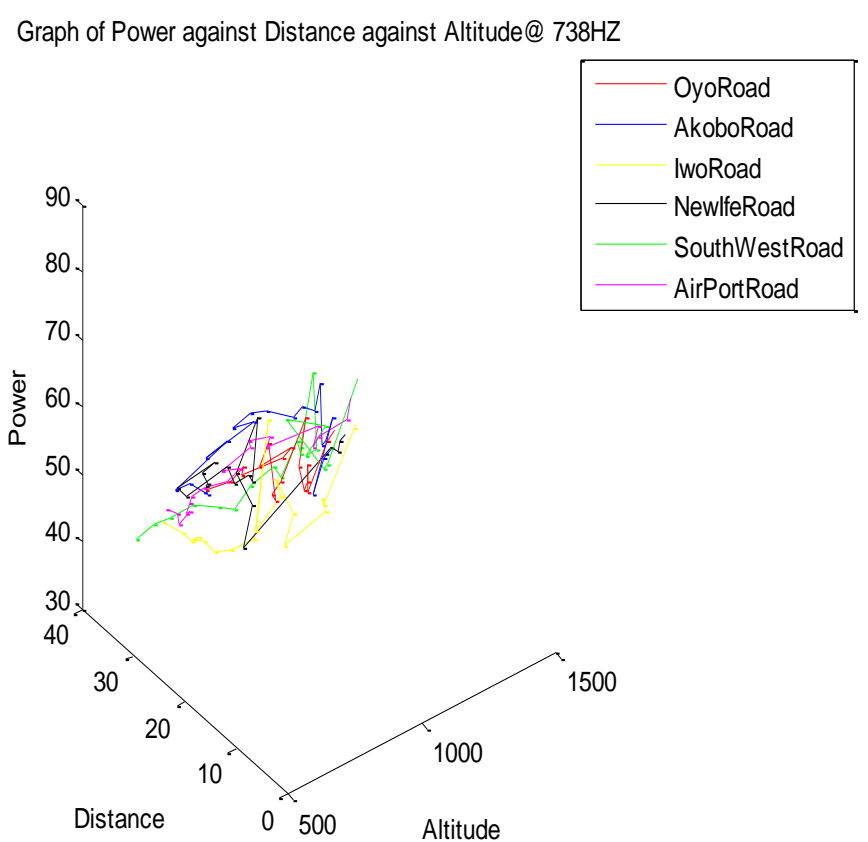

Figure 5. 3-D Plot showing the effect of altitude on the measured signal strength in respect to distance for the $738 \mathrm{~Hz}$ frequency using MATLAB

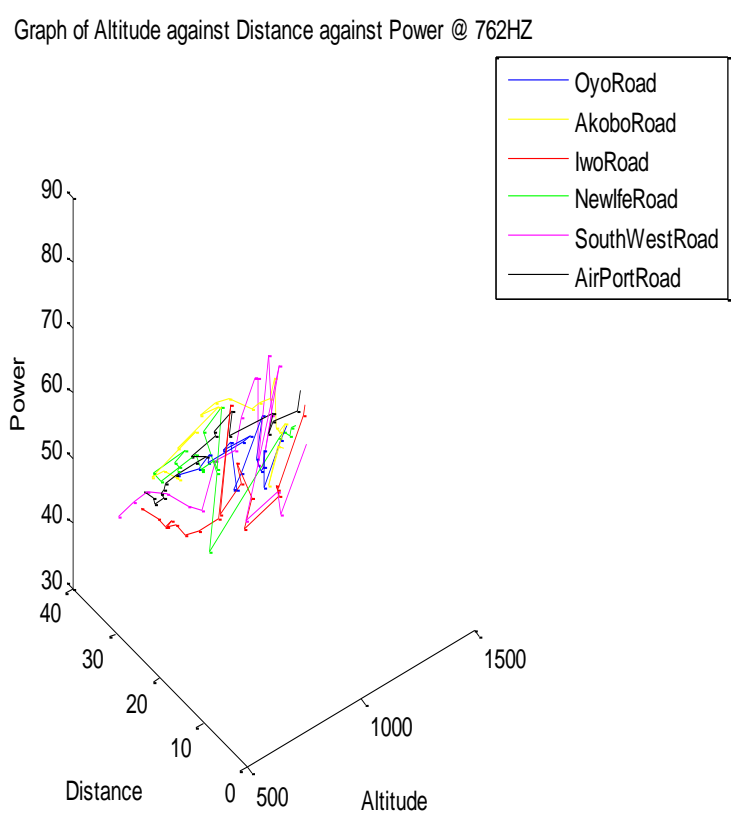

Figure 6. 3-D Plot showing the effect of altitude on the measured signal strength in respect to distance for the $762 \mathrm{~Hz}$ frequency using MATLAB $p$-value $=0.000<0.05$ and also the distance from

Table 7. The power level, altitude, and distance for transmission Station

\begin{tabular}{lccc}
\hline & N & $\begin{array}{c}\text { Correlation } \\
\text { coefficient( } r)\end{array}$ & P value \\
\hline Power Level and Altitude(Ft) & 20 & .618 & .057 \\
Power Level and Altitude(Ft) & 20 & .510 & .132 \\
Power Level and Distance from & 20 & -.873 & .001 \\
$\begin{array}{l}\text { Transmission Station }(\mathrm{km}) \\
\text { Power Level and Distance from }\end{array}$ & 20 & -.790 & .007 \\
Transmission Station((km) & & & \\
\hline
\end{tabular}

Table 8: The effect of power level on the altitude and distance.

\begin{tabular}{llllll}
\hline Parameters & $\begin{array}{l}\text { Std. } \\
\text { Deviation }\end{array}$ & $\begin{array}{l}\text { Std. } \\
\text { Error } \\
\text { Mean }\end{array}$ & $\begin{array}{l}\text { t- } \\
\text { value }\end{array}$ & Df & $\begin{array}{l}\text { P- } \\
\text { value }\end{array}$ \\
\hline PowerLevel/Altitude(Ft) & 80.49019 & 25.45323 & 2.747 & 19 & 0.000 \\
Power Level/ Altitude(Ft) & 80.44766 & 25.43978 & 22.841 & 19 & 0.000 \\
$\begin{array}{l}\text { Power Level/Distance for } \\
\text { Transmission Station(km) }\end{array}$ & 18.55281 & 5.86691 & 6.148 & 19 & 0.000 \\
$\begin{array}{l}\text { Power Level/Distance for } \\
\text { Transmission Station(km) }\end{array}$ & 21.03821 & 6.65287 & 5.108 & 19 & 0.001
\end{tabular}


transmission station have effects on the power level as $p$ value $=0.000<0.05$. Figures 7 to 12 compare the relationship between the signal strength of the two frequencies which reveal how both signal strength reduces progressively as the distance from the transmitter increases. No significant difference was observed for the two frequencies. This show that the transmitting frequencies does not affect the quality of the power of the transmitted signals since the two frequencies are very close in the UHF band.

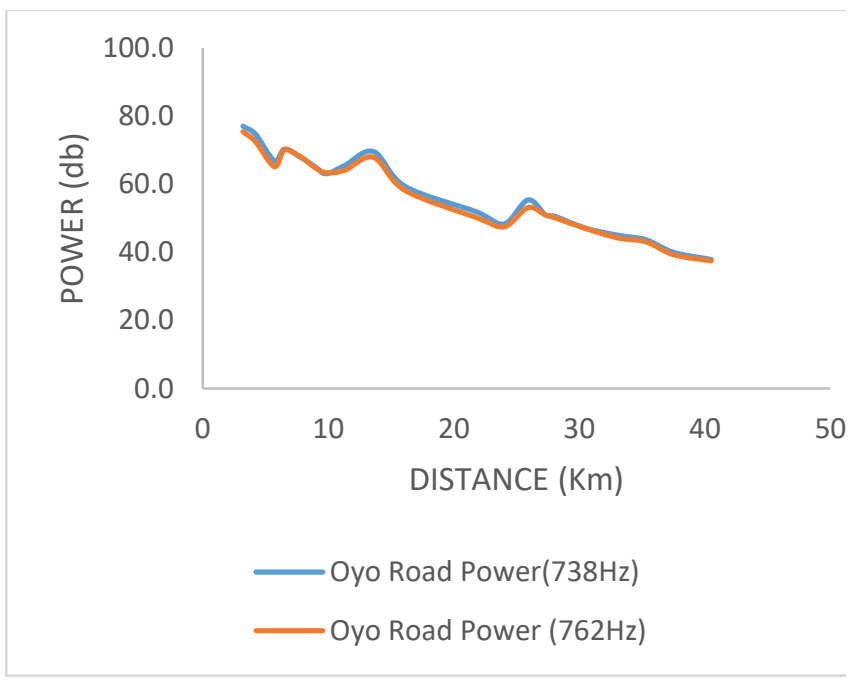

Figure 7. Comparison plot of power level against signal strength along Oyo road for the frequencies 738 and $762 \mathrm{MHz}$.

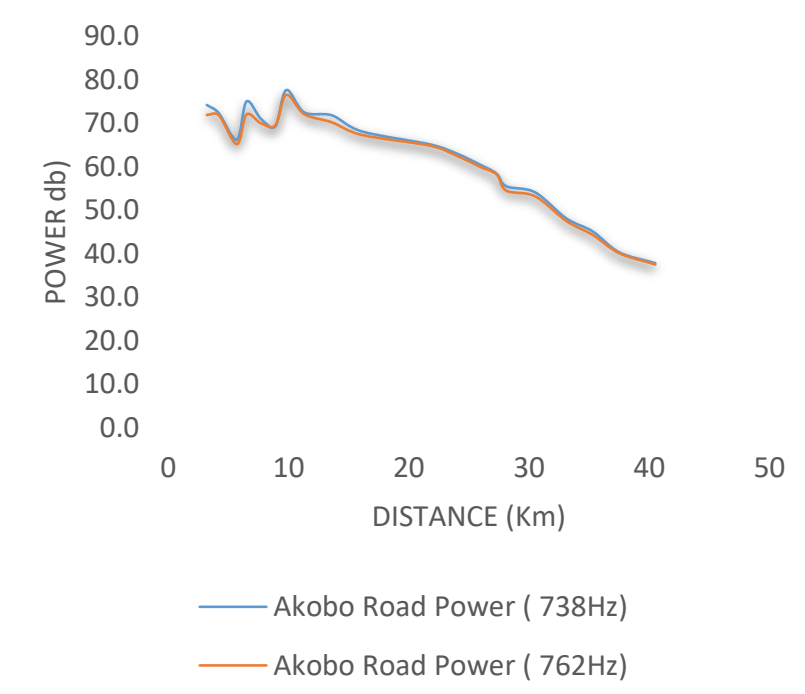

Figure 8. Comparison plot of power level against signal strength along Akobo road for the frequencies 738 and $762 \mathrm{MHz}$

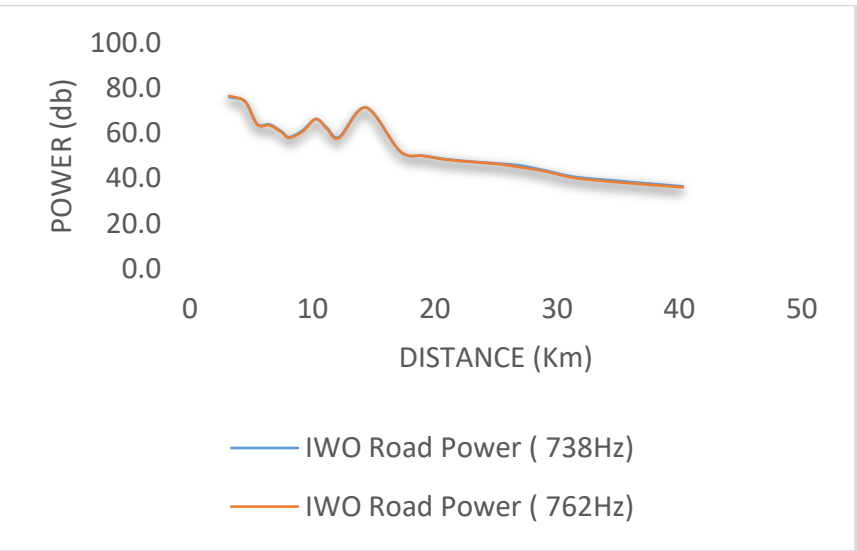

Figure 9. Comparison plot of power level against signal strength along Iwo road for the frequencies 738 and $762 \mathrm{MHz}$.

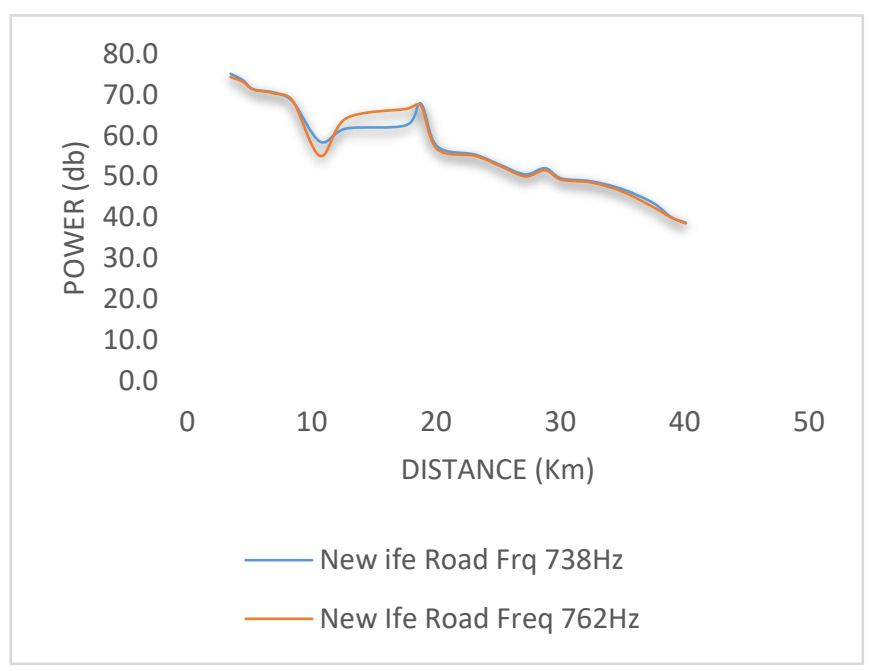

Figure 10. Comparison plot of power level against signal strength along New Ife road for the frequencies 738 and $762 \mathrm{MHz}$.

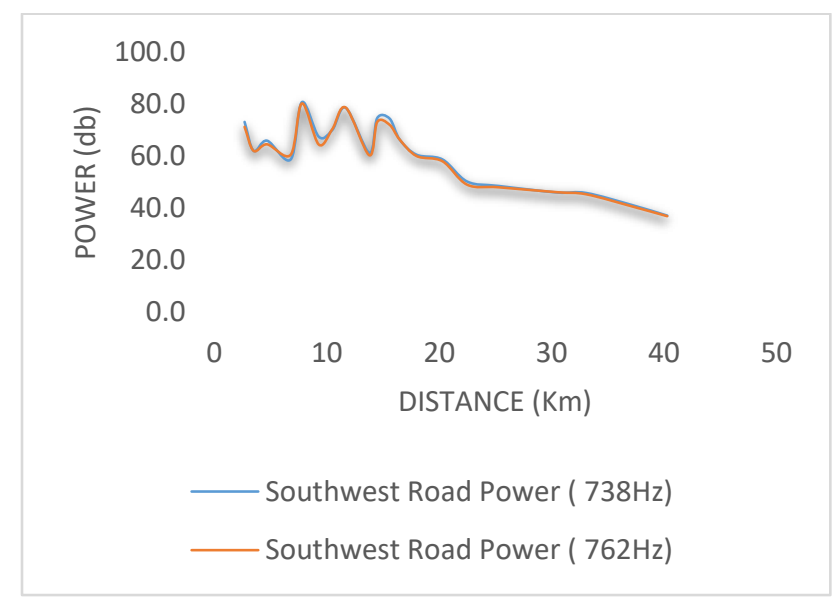

Figure 11. Comparison plot of power level against signal strength along Southwest road for the frequencies 738 and $762 \mathrm{MHz}$. 


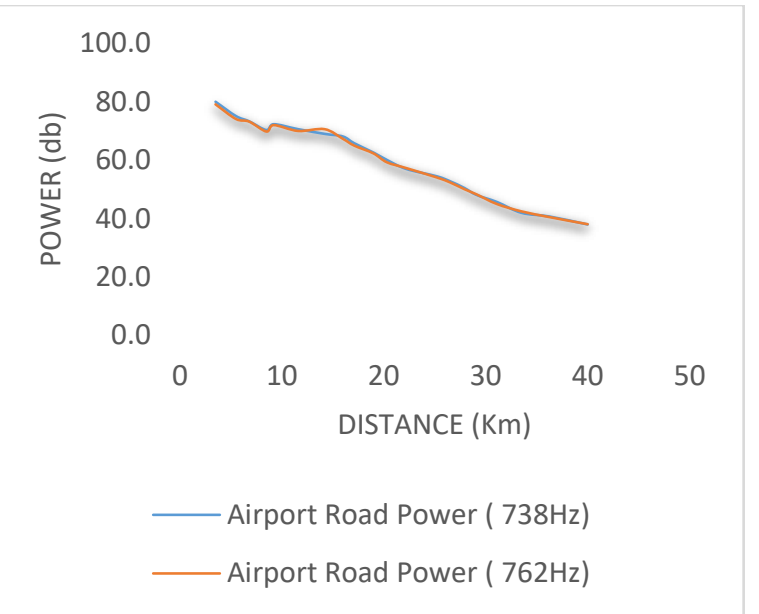

.Figure 12. Comparison plot of power level against signal strength along Airport road for the frequencies 738 and $762 \mathrm{MHz}$

\section{Conclusion}

The results obtained showed that the Startimes transmission parameters are in agreement with the International Telecommunication Union (ITU) standards. It was also observed that there exist an inverse relationship between the distance and signal strength monitored from the transmission station, i.e. the higher the distance, the lower the transmitted power received. It also reveals that there is a direct relationship between the altitudes and the signal strength. The higher the altitude, the higher the received power. The SNR, $M E R$, and BER of the Startimes signal within the $40 \mathrm{~km}$ radius in Ibadan were in agreement with the ITU recommendation for stable signal.

With this finding it could be said that a complete switch over from analogue to digital transmission should not pose serious challenges in Nigeria as the existing DTTV operators operating parameters are well in line with the ITU and ETSI Standards.

\section{References}

Adda , J., \& Ottaviani, M. (2005). The transition to digital television. Economic Policy, 20(4), 160-209.

Ajibola, W. (2015, December). Signal processing of information for digital broadcast. Case study: Nigeria and Kenya. A Thesis Submitted to the Central Ostrobothnia University of Applied Sciences for a Degree Programme in Information Technology.
Balarabe, S. (2013). Digitization of Television Broadcasting in Nigeria Review. World Academy of Science, Engineering and Technology,. International Journal of Social, Behavioral, Educational, Economic, Business and Industrial Engineering, 7(10),, 2767-2771.

BT.2140-7, I.-R. (2014). Transition from analogue to digital terrestrial broadcasting. Geneva: International Telecommunication Unioin.

Collins, G. (2001). Fundamental of Digital Television Transmission. New York: John Wiley \& Sons, Incorporation .

Davis, B. A. (2009). DTV: A case study documenting the transition to digital broadcasting by local television stations. Alabama: University of South Alabama.

Dhok, P., \& Dhanvijay, A. (2015). A Review on Digital Video Broadcasting Terrestrial (DVB-T) Based OFDM System. International Journal of Engineering and Techniques, 1(2), 27-31.

ETSI TR 101 290. (2001). Digital Video Broadcasting: measurement guidelines. London: ETSI.

Fortin, D. (2008). Performance assessment of DVB-T and wireless communication system by means of cross-layer measurements. Tesi di Dottorato, Dipartimento di Ingegneria dell'Informazione.

Gyamfi, E. F. (2015). Technical challenges of the transittion from analog to digital transmission. Ghana: A M.Sc Thesis submitted to the Department of Telecommunication Engineering, Faculty Electrical/Computer Engineering, Kwame Nkrumah University of Science and Technology, Kumasi.

Idachaba, A. (2018). Digitization of Broadcasting in Nigeria: Policy and Implementation. Enugu: Black Belt Konzult Ltd.

Idoko, O. E. (2010). The Challenges of digital television broadcasting in Nigeria. Makurdi Journal of Communication Research.

ITU_R.BT.1306. (2011, 03). Error-correction, data framing, modulation and emission methods for digital terrestrial television broadcasting. Geneva: Electronics Publication.

ITU_R.F.339-8. (2013, Febrary 12). Bandwidths, signal-to-noise ratios and fading allowances in HF fixed and land mobile radiocommunication systems. Retrieved from ITU_R: 
http://www.itu.int/rec/R-REC-TF.1552-0200202-W

Mbatha, B., \& Lesame, Z. (2014). South Africa goes digital: Possible obstacles to the adoption of digital television. Mediterranean Journal of Social Sciences, 5, 89-94.

NBC. (2016, December 23). Nigeria commits to digital television migration in 2017. Retrieved from financial Nigeria: http://www. financialnigeria.com/nigeriacommits-to-digital-television-migration-in2017-news-1023.html

NBC. (2018, April 14). www.nbc.gov.ng. Retrieved from www.nbc.gov.ng: http://www.nbc.gov.ng/pages/signaldistributors

Nkaka , B., \& Mukumbwa, B. (2016). Digital terrestrial television migration challenges - a case study of Zambia. International Journal of Networks and Communications, 6(2), 32-37.
Plum. (2014). Benefits of digital broadcasting: A report for the GSMA. London: Plum Consulting, 88 Kingsway, London, WC2B 6AA, UK.

Satitsamitpong , M., \& Mitomo , H. (2013). An analysis of factors affecting the adoption of digital terrestrial television services in Thailand. International Journal of Managing Public Sector Information and Communication Technologies (IJMPICT).

Seamus, O. L. (2000). Understanding Digital Terrestrial Broadcasting. London: Artech house.

Simon, H. (2000). Communication System. Dehil: Haykin Simon.

Telecom Regulatory Authority of India. (2017). Recommendations on Issues related to Digital Terrestrial Broadcasting in India. New Delhi: Telecom Regulatory Authority of India (TRAI). Retrieved 14-04-2018, from www.trai.gov.in 